\title{
Transcutaneous Electrical Nerve Stimulation to Improve Female Sexual Dysfunction Symptoms: A Pilot Study
}

\author{
Lauren L. Zimmerman, MS* ${ }^{\dagger} \oplus$; Priyanka Gupta, MD*; \\ Florence O'Gara, BSN, MSW ${ }^{\S}$; Nicholas B. Langhals, PhD ${ }^{11}{ }_{\odot}$; \\ Mitchell B. Berger, MD, PhD ${ }^{* *+1} \odot$; Tim M. Bruns, PhD*
}

\begin{abstract}
Objectives: To perform a pilot study using transcutaneous electrical nerve stimulation (TENS) on the dorsal genital nerve and the posterior tibial nerve for improving symptoms of female sexual dysfunction (FSD) in women without bladder problems. We hypothesize that this therapy will be effective at improving genital arousal deficits.

Materials and Methods: Nine women with general FSD completed the study. Subjects received 12 sessions of transcutaneous dorsal genital nerve stimulation (DGNS; $n=6$ ) or posterior tibial nerve stimulation (PTNS; $n=3$ ). Stimulation was delivered for $30 \mathrm{~min}$ at $20 \mathrm{~Hz}$. Sexual functioning was evaluated with the female sexual functioning index (FSFI), and surveys were also given on general health, urological functioning, and the Patients' Global Impression of Change (PGIC) after treatment. Surveys were given before treatment (baseline), after 6 and 12 weeks of treatment, and 6 weeks after the completion of stimulation sessions.

Results: The average total FSFI score across all subjects significantly increased from $15.3 \pm 4.8$ at baseline to $20.3 \pm 7.8$ after six sessions, $21.7 \pm 7.5$ after 12 sessions, and $21.3 \pm 7.1$ at study completion ( $p<0.05$ for all time points). Increases were observed in both DGNS and PTNS subjects. Significant FSFI increases were seen in the subdomains of lubrication, arousal, and orgasm, each of which is related to genital arousal. Bladder and general health surveys did not change across the study. PGIC had a significant increase.
\end{abstract}

Conclusions: This study provides evidence that transcutaneous stimulation of peripheral nerves has the potential to be a valuable therapeutic tool for women with FSD.

Keywords: Dorsal genital nerve, electrical stimulation, female sexual dysfunction, genital arousal, posterior tibial nerve

Conflict of Interest: None.

\section{INTRODUCTION}

Female sexual dysfunction (FSD) affects $40-45 \%$ of adult women and is a difficult condition to diagnose and treat $(1,2)$. Low genital arousal and poor lubrication affects between 8 and $28 \%$ of women and orgasm difficulties affect $16-25 \%(2,3)$, and can be due to underactive neural or vascular activity in the genitals, urological problems, or other pelvic floor dysfunctions (4). Low sexual desire, or interest, affects $9-39 \%$ of women $(2,5)$, and may be due to hyperactivity in prefrontal areas of the brain (4). Women are more likely to have FSD as they age, and women often have more than one form of FSD (2). An active and satisfying sex life is widely regarded not only as desirable but as a sign of emotional and physical health. The sexually disinterested person with arousal difficulties is made to feel deficient, dissatisfied, or dysfunctional (6). Medical providers and therapists are challenged by treatment as there are multiple possible contributors and different forms of FSD. Hormone therapy can be effective for genital and desire dysfunctions, but is not recommended for all individuals and is typically not recommended for long-term treatment (7). Flibanserin, a recently FDA-approved drug, has some success in increasing sexual desire but does not impact genital arousal $(8,9)$. Sildenafil has occasionally been reported to
Address correspondence to: Tim M. Bruns, PhD, Department of Biomedical Engineering, University of Michigan, 2800 Plymouth Road, NCRC B10-A169, Ann Arbor, MI 48109, USA. Email: bruns@umich.edu

* Department of Biomedical Engineering, University of Michigan, Ann Arbor, MI, USA;

${ }^{\dagger}$ Biointerfaces Institute, University of Michigan, Ann Arbor, MI, USA;

₹ Department of Urology, University of Michigan, Ann Arbor, MI, USA;

§ Sexual Health Counseling, University of Michigan, Ann Arbor, MI, USA;

" Department of Surgery, Plastic Surgery Section, University of Michigan, Ann Arbor, MI, USA;

** Department of Obstetrics \& Gynecology, University of Michigan, Ann Arbor, $\mathrm{MI}, \mathrm{USA}$; and

${ }^{+\dagger}$ Obstetrics \& Gynecology, Main Line Health, Wynnewood, PA, USA

For more information on author guidelines, an explanation of our peer review process, and conflict of interest informed consent policies, please go to http:// www.wiley.com/WileyCDA/Section/id-301854.html

${ }^{1}$ Current Address: Nicholas B. Langhals, National Institute of Neurological Disorders and Stroke, National Institutes of Health, Bethesda, MD, USA.

Mitchell B. Berger and Tim M. Bruns are co-senior authors.

Source(s) of financial support: This study was funded in part by a grant from the Michigan Institute for Clinical and Health Research (MICHR), which is funded by the National Center for Advancing Translational Sciences (NCATS) of the National Institutes of Health (Grants UL1TR000433 and UL1TR002240). 
improve genital arousal (10), but results are inconsistent and frequently present with mild to moderate side-effects such as headaches, flushing, rhinitis, and nausea (11). There is a need for an effective treatment for women who have genital arousal deficiencies without concurrent side-effects.

Peripheral neuromodulation therapies have been implemented for patients with bladder dysfunction for decades. Sacral neuromodulation (SNM) involves the surgical implantation of a stimulation system, with an electrode near the S3 sacral foramen delivering continuous stimulation (12). Dorsal genital nerve stimulation (DGNS) is typically delivered transcutaneously above the clitoris and lateral to the labia majora in women (13-15), though percutaneous electrodes may also be used (16). The dorsal genital nerve is a distal branch of the pudendal nerve, which is stimulated centrally with SNM. Percutaneous tibial nerve stimulation (PTNS) is a treatment where patients receive $30 \mathrm{~min}$ of electrical stimulation a week for 12 weeks with periodic maintenance sessions thereafter $(17,18)$, though benefits have been observed after as few as six sessions (19). Stimulation is delivered via a percutaneous needle placed at the tibial nerve near the ankle, but cutaneous stimulation with transcutaneous electrical nerve stimulation (TENS) electrodes has also shown efficacy in some studies (20-22). The underlying mechanisms of these neuromodulation therapies are not well understood, with evidence suggesting inhibition at spinal and/or supra-spinal levels affecting efferent control over bladder storage and emptying $(19,23)$.

In clinical studies in which patients received neuromodulation treatment for bladder dysfunction, significant improvements in sexual functioning as evaluated with the Female Sexual Function Index (FSFI) were noted for SNM (24-27) and PTNS (28-30) therapies. While bladder dysfunction has a known negative effect on sexual function $(31,32)$, improvements in sexual functioning were found to be independent from improvements in bladder functioning $(25,30)$, indicating that the neuromodulation may have a direct impact on genital arousal. No studies have evaluated the effects of peripheral nerve stimulation specifically on patients with FSD without an underlying urological condition.
The goal of this pilot study was to evaluate weekly skin-surface TENS of the dorsal genital nerve and the posterior tibial nerve for improving sexual function in women with FSD and no clinically diagnosed bladder problems.

\section{METHODS}

Approval for this study was obtained from the Michigan Medicine Institutional Review Board prior to initiation (study number HUM00101713). Participants were recruited through Michigan Medicine sexual health practices, gynecology clinics, and an online University of Michigan health research portal (umhealthresearch.org). This study was registered at clinicaltrials. gov under identifier NCT02692417.

In a phone call with a study coordinator, subjects were screened for study eligibility. All subjects were 18 years or older cis-gender women, neurologically stable, and sexually active at least once a month. The short-form FSFI- 6 was used to screen for FSD, with scores below 19 required for inclusion (33). The specific type or types of FSD that each participant had was not identified as part of the screening process. Women who were pregnant or planning pregnancy, had clinically diagnosed bladder dysfunction or pelvic pain, previous pelvic surgery, experience with electrical stimulation for bladder or sexual problems, recent use of TENS on their pelvis, back, or legs, had an implanted pacemaker, defibrillator, spinal cord stimulator, or other nerve stimulator, or were taking any investigational drug were excluded from the study. All subjects provided written informed consent. A pregnancy test was also performed at the first session to confirm nongravidity if the subjects were premenopausal and had not had a hysterectomy. Menopause status was not specifically tracked across participants. The intended sample size of this study was 20 subjects, with 10 subjects in each study group, similar to other neuromodulation pilot studies $(13,14,34)$. As described below, a smaller sample size was reached due to challenges in subject recruitment and retention.

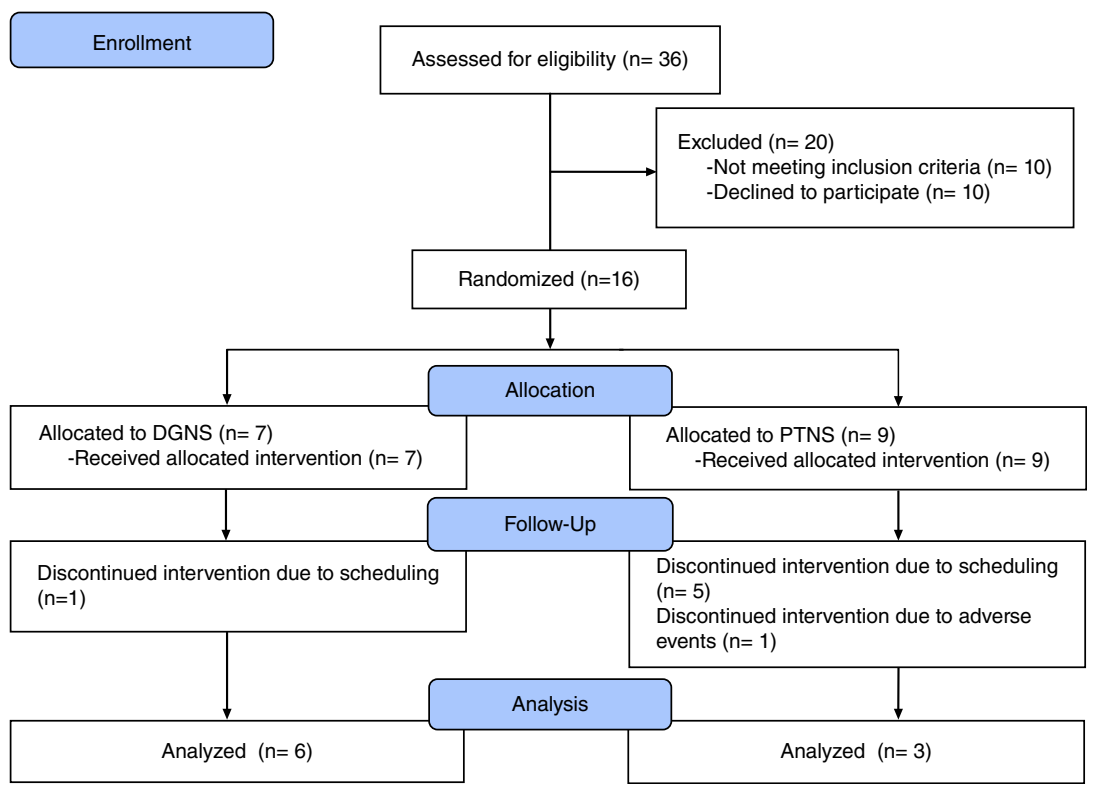

Figure 1. Study CONSORT flow diagram. [Color figure can be viewed at wileyonlinelibrary.com] 
Table 1. Patient Demographics.

$\begin{array}{ll}\text { Category } & \text { All enrolled participants } \\ \text { Total } & 16 \\ \text { Age (years) } & 40.9 \pm 15.0 \\ \text { BMI }\left(\mathrm{kg} / \mathrm{m}^{2}\right) & 26.8 \pm 4.4 \\ \text { Race/Ethnicity } & \\ \quad \text { White } & 10(67 \%) \\ \text { Black } & 1(7 \%) \\ \text { Asian or Pacific Islander } & 1(7 \%) \\ \text { Other } & 3(20 \%) \\ \text { Relationship status } & \\ \quad \text { Single } & 2(13 \%) \\ \quad \text { Nonmarried relationship } & 3(20 \%) \\ \quad \text { Married } & 10(67 \%) \\ \text { On prescription antidepressant } & 6(40 \%) \\ \text { Baseline FSFI } & 17.1 \pm 5.0 \\ \text { Baseline SF-36 } & 83.1 \pm 11.6 \\ \text { Baseline AUASI } & 6.4 \pm 5.3\end{array}$

$\begin{array}{ll}\text { PTNS completed } & \text { DGNS completed } \\ 3 & 6 \\ 37.3 \pm 19.1 & 50.7 \pm 11.0 \\ 28.4 \pm 5.0 & 26.3 \pm 4.9 \\ 2(67 \%) & 6(100 \%) \\ 1(33 \%) & 0(0 \%) \\ 0(0 \%) & 0(0 \%) \\ 0(0 \%) & 0(0 \%) \\ 0(0 \%) & 1(17 \%) \\ 1(33 \%) & 0(0 \%) \\ 2(67 \%) & 5(83 \%) \\ 2(67 \%) & 3(50 \%) \\ 15.2 \pm 5.3 & 15.5 \pm 4.6 \\ 87.9 \pm 4.4 & 80.5 \pm 9.0 \\ 9.3 \pm 6.4 & 4.5 \pm 2.9\end{array}$

At the first stimulation session, patients were randomized into one of two study groups, DGNS or PTNS. Randomization was accomplished using a random-number table and block size of two. Allocation assignment was performed using sequentially numbered, opaque sealed envelopes, which were opened in the presence of the subjects. Subjects received skin-surface stimulation with a TENS unit (Empi Select, DJO Global, Vista, CA, USA). Electrodes were 1.25-inch round neurostimulation electrodes (ValuTrode Fabric CF3200, Axelgaard Manufacturing Co., Ltd., Fallbrook, CA, USA). For DGNS participants, each electrode was placed on either lateral side of the clitoris (15). For PTNS participants, electrodes were placed just above the medial malleolus and the ipsilateral calcaneus $(21,22)$. Stimulation for both arms was applied at $20 \mathrm{~Hz}$, as is typical for PTNS (35). For both DGNS and PTNS subjects, starting from a low amplitude, current was increased until the participant expressed discomfort, and then reduced to a comfortable level, or a maximal level of $60 \mathrm{~mA}$ was reached. Subsequently, stimulation was applied using that amplitude for $30 \mathrm{~min}$, at $20 \mathrm{~Hz}$.

Participants completed a total of 12 stimulation sessions $(12,17,35)$. Our goal was to schedule sessions on consecutive weeks for the duration of participation. However, scheduling conflicts, holidays, and other events led to variations in intrasession intervals across subjects. Participants were compensated for their time.

Patients completed a series of validated clinical surveys as outcome measures at baseline, after 6 stimulation sessions, after 12 stimulation sessions, and 6 weeks after the final session. At all survey intervals, participants completed the full FSFI (36), the short-form 36-question (SF-36) quality of life survey (37), and the 6-question American Urological Association Symptom Index (AUASI) bladder symptom index (38). At the 6-week and later survey intervals participants also completed the one-question Patients' Global Impression of Change (PGIC) (39). All surveys were completed and stored through a secure online portal (REDcap) (40).

Comparisons between FSFI, SF-36, AUASI, and PGIC scores at different time points were analyzed with related-samples Wilcoxon signed rank tests with a significance level of 0.05 . Tests were run with DGNS and PTNS arms separately as well as pooled together. Where appropriate, values are presented as mean \pm standard deviation.

\section{RESULTS}

Sixteen subjects were enrolled in the study (Fig. 1). Seven subjects dropped out of the study during intervention, due to scheduling conflicts $(n=6)$ and an adverse event $(n=1$; described below). Of the nine subjects that completed the study, the average age was $46.2 \pm 14.5$, with a minimum age of 23 and maximum of 66 (Table 1). One subject who was enrolled but did not receive stimulation did not complete the demographics survey.

Stimulation was not always delivered in exact 1-week intervals. The average interval between sessions was $12.5 \pm 10.3$ days. The stimulation current amplitude that was delivered ranged from 2.5 to $60.0 \mathrm{~mA}$. Stimulation was delivered at $24.3 \pm 18.6 \mathrm{~mA}$ for DGNS subjects, and $60.0 \pm 0.0 \mathrm{~mA}$ for PTNS subjects.

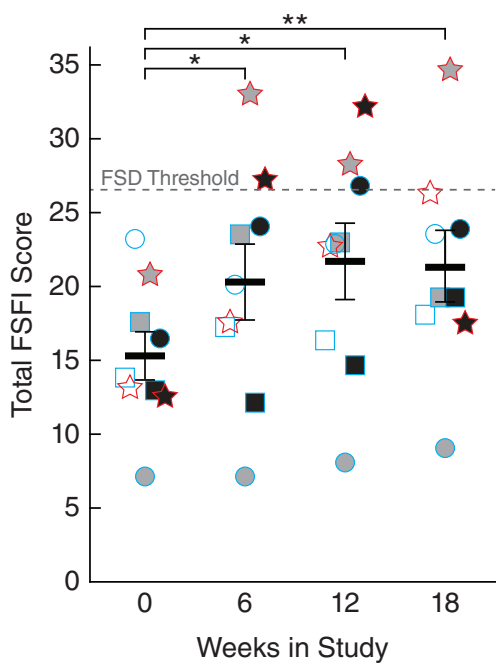

Figure 2. Average total FSFI score for all subjects (PTNS and DGNS) at each survey time point. Error bars give standard error of the mean. Significant improvement from baseline occurred at each time point. Individual icons are unique for each participant, with PTNS participants indicated with stars and DGNS participants indicated with circles and squares. White, gray, and black shading inside of each icon further distinguishes between different subjects. Within each study week, icon order from left to right indicates study participation order $\left(^{*} p<0.05\right.$; ${ }^{* *} p<0.01$ ). [Color figure can be viewed at wileyonlinelibrary.com] 


\section{Desire}
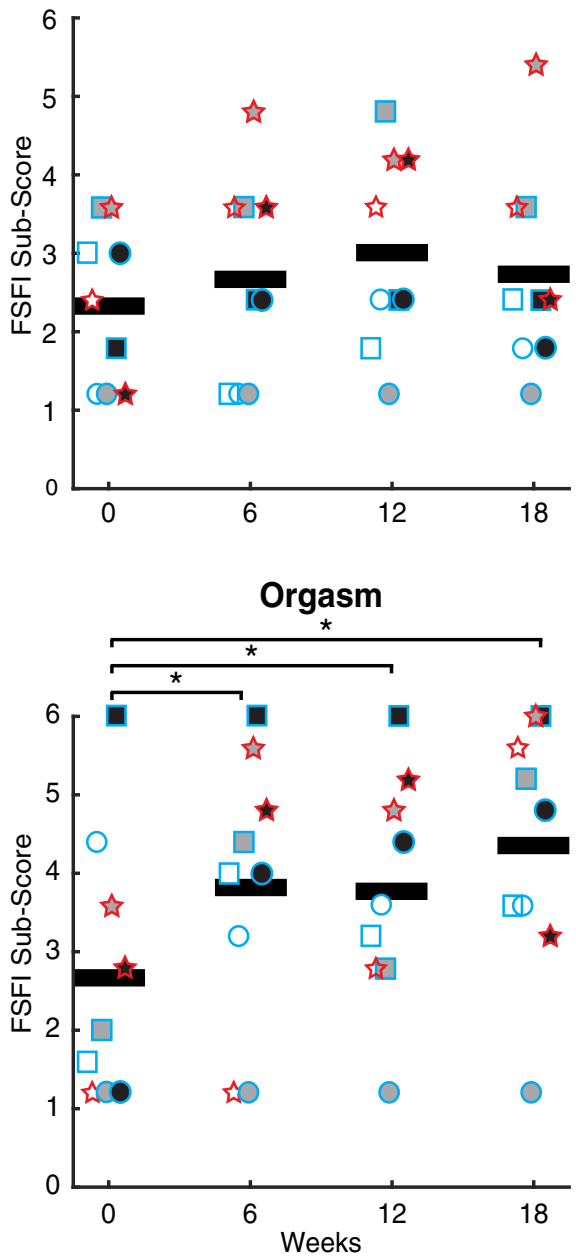

Arousal

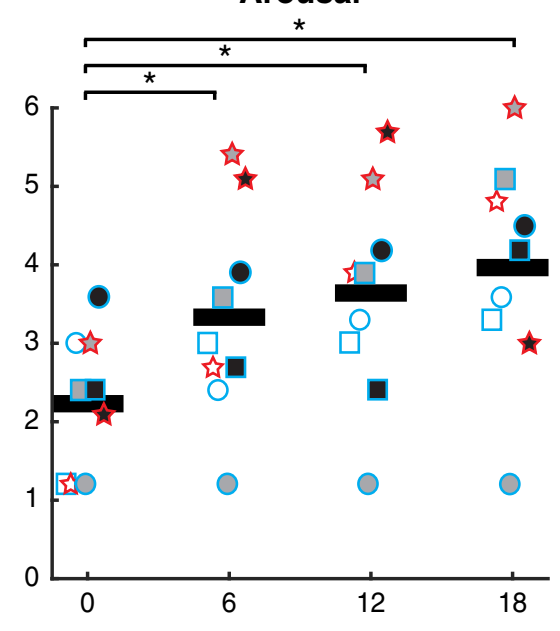

Satisfaction

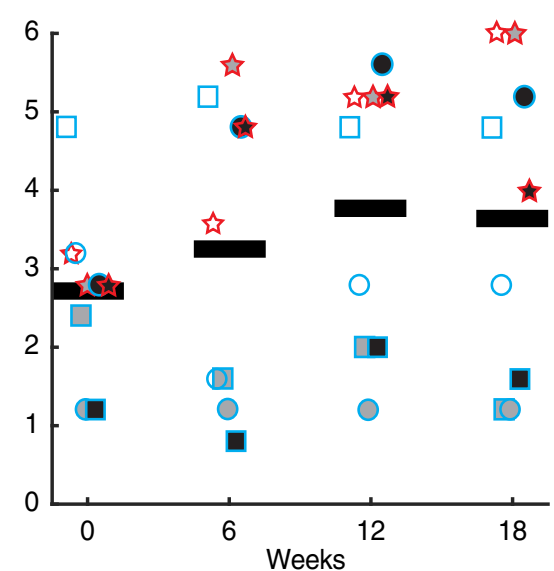

Lubrication

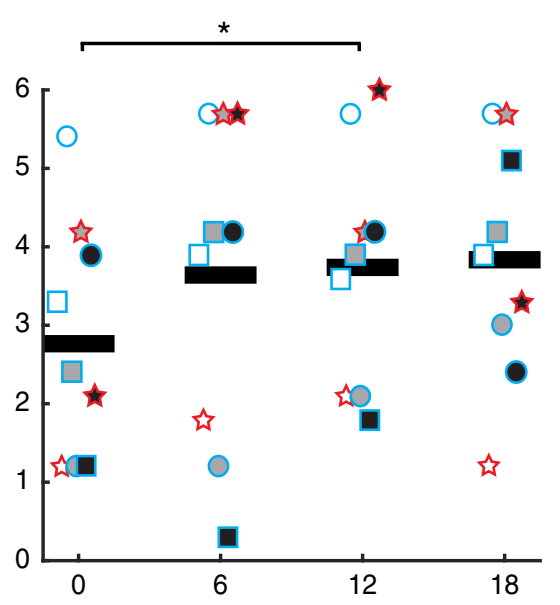

Pain

Figure 3. Individual FSFI subdomain scores for all subjects. The pooled mean is given by the horizontal bar. Individual icons are unique for each participant, following the convention in Fig. 2. Within each study week, icon order from left to right indicates study participation order $\left({ }^{*} p<0.05\right)$. [Color figure can be viewed at wileyonlinelibrary.com]

All women began the study with an FSFI total score below the clinical cut-off for diagnosing FSD (26.55) (41), with an average initial score of $15.3 \pm 4.8$. Overall sexual function significantly increased at 6, 12, and 18 weeks from baseline (Fig. 2, Table 2). Three of the nine subjects (33.3\%) reached an FSFI score above the clinical cut-off for FSD, and another participant scored just below the threshold (26.4). Four subjects (1 DGNS, 3 PTNS) had a clinically relevant increase in their FSFI score, with an improvement of at least $50 \%$. Arousal and orgasm FSFI subscores had significant improvements at 6,12 , and 18 weeks from baseline (Fig. 3, Table 2) Lubrication FSFI subscores had a significant improvement at 12 weeks over baseline (Fig. 3, Table 2). Each of the other FSFI subscores (desire, satisfaction, and pain) had nonsignificant increases in their scores (Fig. 3, Table 2).

Changes in FSFI scores were not related to variations in the intervals between stimulation sessions. The FSFI percent increase had no relationship with average stimulation session intervals at 12 weeks $\left(y=-0.077 \mathrm{x}+40.58, R^{2}=0.0139, p=0.78\right)$ or at 18 weeks $\left(y=-0.1309 x+57.97, R^{2}=0.026, p=0.70\right)$.

Overall, participants perceived an improvement in sexual function, as PGIC scores were $3.3 \pm 2.0$ at 6 weeks $(3.0=$ "a little better"), $4.0 \pm 1.9$ at 12 weeks $(4.0=$ "somewhat better"), and $4.1 \pm 1.9$ at 18 weeks. These scores are each significantly different from a PGIC score of $1.0(p=0.018, p=0.011$, and $p=0.011$, respectively), which would indicate "no change or worse." The three women (2 PTNS, 1 DGNS) who achieved FSFI scores above the FSD clinical cutoff scored either a 5 ("moderately better") or 6 ("better") at each time point. Overall quality of health scores from the SF-36 remained generally stable across the study duration. The SF-36 category of role limitations due to physical health improved from $88.9 \%$ pretreatment to $97.2 \%$ at the 18-week time point across all subjects. Also the SF-36 category emotional well-being showed a significant worsening from $80.0 \%$ pretreatment to $74.2 \%$ at week $6(p=0.042)$ for DGNS subjects. Participant's bladder functioning, as scored by the AUASI, did not show significant change across all subjects across the study time points, except for the domain of nocturia. There was a significant $25.0 \%$ reduction in nocturia symptoms ( $p=0.046)$ from baseline $(1.78 \pm 1.30)$ to the 18 -week timepoint $(1.33 \pm 1.22)$.

Subjects were given the opportunity to refrain from answering questions. The unanswered questions were scored as a 0 , which 
Table 2. Average FSFI Scores Across All Subjects As Well As Across Two Subject Groups, With Standard Deviation in ().

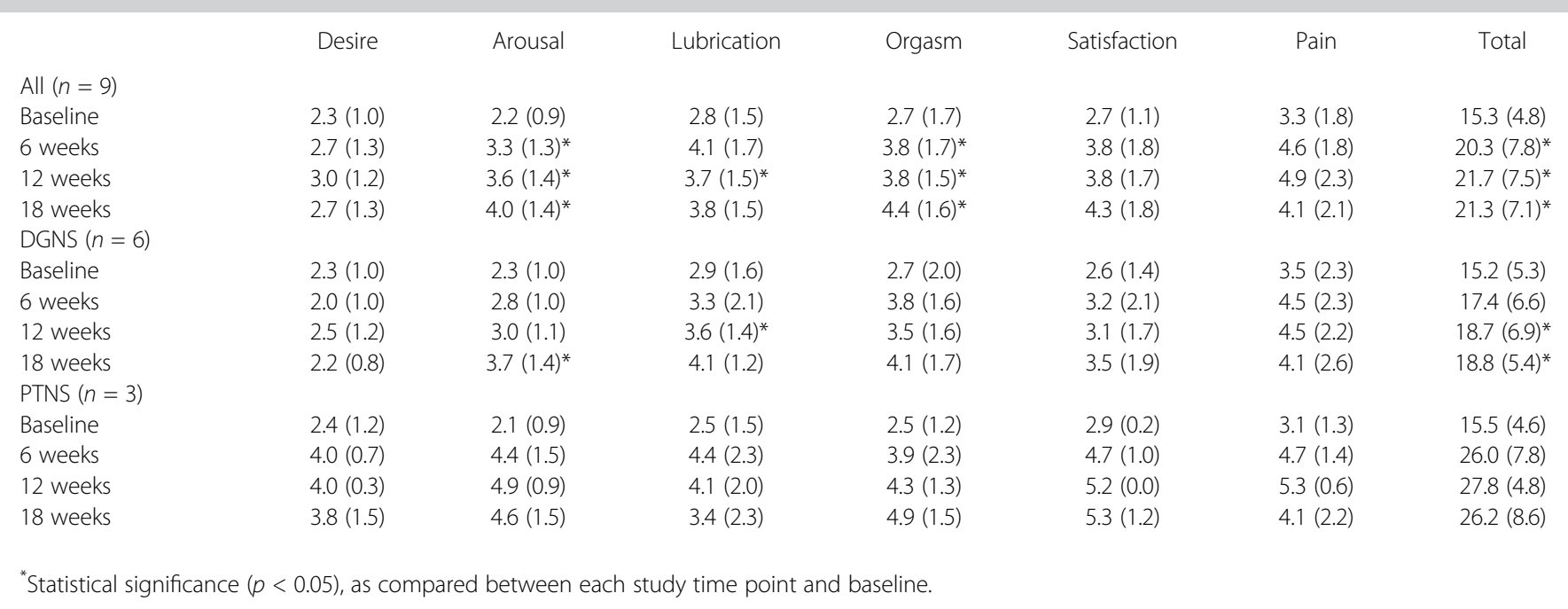

negatively affected FSFI scoring. Three DGNS subjects (blue/white square, blue/gray square, and blue/black square in Figs. 2 and 3) refrained from answering questions about pain. Two of those subjects (blue/gray square and blue/black square) also refrained from answering some of the questions about satisfaction in two surveys. One of those subjects (blue/black square) also refrained from answering some of the questions about lubrication in one survey. One subject (red/black star) reported that between weeks 12 and 18 surveys, she was diagnosed with a severe pelvic infection from Escherichia coli. She indicated that this unrelated event would negatively impact her 18-week survey, as seen by declines in her scores from weeks 12 to 18 , particularly in pain (Fig. 3).

One participant receiving PTNS withdrew from the study after three sessions after feeling sciatic nerve pain during stimulation. The subject had a history of sciatic pain. Aggravation reemerged after both lowering the amplitude of current delivered and switching the stimulation location to the alternate leg.

\section{DISCUSSION}

In this study, we demonstrate the feasibility of transcutaneous stimulation as a treatment for genital arousal disorders in women. Significant improvements were achieved in the areas of arousal, lubrication, and orgasm (Fig. 3, Table 2), leading to overall better sexual functioning (Fig. 2). These domains are each related to genital arousal. Subjects reported the highest sexual functioning at 12 weeks into the study, after having received all stimulation sessions. A slight decrease in overall FSFI scores occurred at 18 weeks, after a 6-week washout period without stimulation, suggesting that maintenance sessions may be beneficial. Maintenance sessions are common for patients receiving PTNS for bladder symptoms, with patients receiving a stimulation session every 2-4 weeks after the initial 12 weeks of therapy to maintain the therapeutic benefits (42). The subjects in the PTNS arm had a greater improvement in sexual functioning (Table 2), but the imbalance of subjects in each arm makes it difficult to perform any statistical comparisons. As $100 \%$ of PTNS subjects increased their total FSFI score by at least $50 \%$, compared to $16.7 \%$ of the DGNS group, it is possible that PTNS is a more effective treatment modality, although further studies with larger sample sizes are needed. Two PTNS subjects commented that they planned to purchase their own TENS equipment to continue treatment at home after study completion.

Across all subjects, the average total of FSFI score increased by 6.4 (Table 2). This increase is comparable to or greater than recent clinical trials studying other treatments for women with desire and/or arousal subtypes of FSD. In the BEGONIA trial investigating FDA-approved flibanserin for hypoactive sexual desire, the treatment group total FSFI score improved by 5.3 against the placebo group increase of 3.5 (8), while a study of bremelanotide saw a total FSFI increase of 4.4 in the most effective treatment group against a placebo increase of 1.9 (43). Clinical studies of neuromodulation, which presumably would benefit genital arousal disorders over hypoactive sexual desire, have reported a total FSFI score increase of 6.5 in a group of patients receiving PTNS for OAB (30) and 4.3 in a group of patients with SNM implants (44), with other SNM and PTNS neuromodulation studies reporting even smaller FSFI increases (range: 2.1-3.3) (24-27,29). That our FSFI increase was comparable to or higher than neuromodulation studies using invasive stimulation electrodes suggests that noninvasive transcutaneous stimulation can yield effective results. A larger, controlled study is needed to verify that we did not have an overall placebo effect higher than those reported in the flibanserin and bremelanotide studies. The nonsignificant increases in nongenital arousal related FSFI subdomains (desire, satisfaction, and pain; Fig. 3, Table 2) could indicate that stimulation led to benefits in these areas, either directly or indirectly through improvements in genital arousal, or those increases may relate to any general placebo effects that occurred in our study.

These results provide further evidence that the improvements to sexual functioning seen in neuromodulation studies for bladder dysfunction are a direct result of the therapy, as opposed to a secondary result from treated bladder symptoms. Peripheral nerve stimulation could be used as a clinical tool to treat women with genital arousal deficiencies. Women who may benefit from this treatment have a variety of potential underlying conditions, including diabetes-related complications, neurological conditions such as multiple sclerosis and 
spinal cord injury, and side-effects of hormonal changes, trauma, and even childbirth $(4,5,45,46)$. A potential mechanism in the observed improvements in genital arousal in our study is an increase in pelvic blood flow, as has been modeled by preclinical studies investigating similar stimulation techniques $(47,48)$, however more research is needed.

An important limitation in this study is the lack of a control. As the results are based on patient-reported outcomes, the impact of a placebo effect could be considerable. Neither the researchers nor the subjects were blinded. There were challenges in recruitment for the study, but more notably in retention. Two primary factors were a need for weekly stimulation sessions during normal business hours and the location of the clinical research center, which required a car or bus to reach. Six of the seven subjects who discontinued the study were in the PTNS arm (Fig. 1), leading to an unequal distribution of subjects. Once enrolled, it was also difficult to schedule subjects every week, so most did not complete the study in the expected 18 weeks. This was due to both patient scheduling conflicts as well as clinician availability. Although the stimulation session intervals often differed from standard PTNS clinical practice for bladder symptoms, no effect on our results was observed. Finally, skin-surface transcutaneous stimulation was utilized, and though it has been shown to be effective clinically $(15,49,50)$, it is less specific than percutaneous needles.

Future studies with sham or placebo controls, as have been completed for bladder care, are necessary to confirm the efficacy of this treatment modality (17). In addition, percutaneous stimulation could be used for more accurate recruitment of target nerves.

\section{CONCLUSION}

This study provides further evidence that improvements seen in the sexual functioning of women receiving neuromodulation treatment for bladder dysfunction were independent of improvements in bladder symptoms, and that stimulation can have a direct impact on sexual arousal. Improvements were primarily seen in genital arousal components of sexual functioning, including lubrication, arousal, and orgasm. Thus, this pilot study demonstrates the feasibility of using transcutaneous neuromodulation of peripheral nerves to treat symptoms of FSD.

\section{Acknowledgements}

We are very appreciative of the time given by our study participants. We also thank Julie Tumbarello, Nina Dutta, Nicole Honey, and Abigail Teitelbaum for their help in study coordination and execution. This article was prepared while Dr. Langhals was employed at The National Institute of Neurological Disorders and Stroke. The opinions expressed in this article are the authors' own and do not reflect the view of the National Institutes of Health, the Department of Health and Human Services, or the United States government.

\section{Authorship Statements}

Dr. Mitchell Berger, Dr. Tim Bruns, Dr. Nick Langhals, and Florence O'Gara designed the study. Drs. Mitchell Berger, Tim Bruns, and Nick Langhals obtained funding for the study. Drs. Mitchell Berger and Priyanka Gupta performed stimulation sessions. Dr. Tim Bruns and Lauren Zimmerman analyzed the data. Dr. Mitchell Berger, Dr. Tim Bruns, and Lauren Zimmerman drafted the manuscript. All authors reviewed and approved the final manuscript.

\section{How to Cite this Article:}

Zimmerman L.L., Gupta P., O'Gara F., Langhals N.B., Berger M.B., Bruns T.M. 2018. Transcutaneous Electrical Nerve Stimulation to Improve Female Sexual Dysfunction Symptoms: A Pilot Study. Neuromodulation 2018; 21: 707-713

\section{REFERENCES}

1. Laumann EO, Paik A, Rosen RC, Page P. Sexual dysfunction in the United States: Prevalence and predictors. JAMA 1999;281:537-545.

2. Shifren JL, Monz BU, Russo PA, Segreti A, Johannes CB. Sexual problems and distress in United States women: prevalence and correlates. Obstet Gynecol 2008, 112:970-978.

3. Lewis RW, Fugl-Meyer KS, Corona G et al. Definitions/Epidemiology/Risk factors for sexual dysfunction. J Sex Med 2010;7:1598-1607.

4. Levin RJ, Both S, Georgiadis J, Kukkonen T, Park K, Yang CC. The physiology of female sexual function and the pathophysiology of female sexual dysfunction (Committee 13A). J Sex Med 2016;13:733-759.

5. Clayton AH, Valladares Juarez EM. Female sexual dysfunction. Psychiatr Clin North Am 2017;40:267-284.

6. Center for Drug Evaluation and Research \& U.S. Food and Drug Administration. The voice of the patient: female sexual dysfunction. http://www.fda.gov/ downloads/Drugs/NewsEvents/UCM453718.pdf

7. Allahdadi KJ, Tostes RCA, Webb RC. Female sexual dysfuction: Therapeutic options and experimental challenges. Cardiovasc Hematol Agents Med Chem 2009;7:260-269.

8. Katz M, Derogatis LR, Ackerman R et al. Efficacy of flibanserin in women with hypoactive sexual desire disorder: Results from the BEGONIA trial. J Sex Med 2013;10:1807-1815.

9. Portman DJ, Brown L, Yuan J, Kissling R, Kingsberg SA. Flibanserin in postmenopausal women with hypoactive sexual desire disorder: results of the PLUMERIA study. J Sex Med 2017;14:834-842.

10. Cavalcanti AL, Bagnoli VR, Fonseca ÂM et al. Effect of sildenafil on clitoral blood flow and sexual response in postmenopausal women with orgasmic dysfunction. Int J Gynecol Obstet 2008;102:115-119.

11. Basson R, Mclnnes R, Smith MD, Hodgson G, Koppiker N. Efficacy and safety of sildenafil citrate in women with sexual dysfunction associated with female sexual arousal disorder. J Womens Health Gend Based Med 2002;11:367-377.

12. Gupta P, Ehlert MJ, Sirls LT, Peters KM. Percutaneous tibial nerve stimulation and sacral neuromodulation: an update. Curr Urol Rep 2015;16:4-9.

13. Vodusek DJ, Light JK, Libby JM. Detrusor inhibition induced by stimulation of pudendal nerve afferents. Neurourol Urodyn 1986;5:381-389.

14. Previnaire JG, Soler JM, Boileau G et al. Short-term effect of pudendal nerve electrical stimulation on destrusor hyperreflexia in spinal cord injury patients: importance of current strength. Int Med Soc Paraplegia 1996;34:95-99.

15. Opisso E, Borau A, Rijkhoff NJM. Subject-controlled stimulation of dorsal genital nerve to treat neurogenic detrusor overactivity at home. Neurourol Urodyn 2013; 32:1004-1009.

16. Goldman HB, Amundsen CL, Mangel J et al. Dorsal genital nerve stimulation for the treatment of overactive bladder symptoms. Neurourol Urodyn 2008;27: 499-503.

17. Peters KM, Carrico DJ, Perez-marrero RA et al. Randomized trial of percutaneous tibial nerve stimulation versus sham efficacy in the treatment of overactive bladder syndrome: results from the SUmiT trial. J Urol 2010;183:1438-1443.

18. Moossdorff-Steinhauser HFA, Berghmans B. Effects of percutaneous tibial nerve stimulation on adult patients with overactive bladder syndrome: a systematic review. Neurourol Urodyn 2013;32:206-214.

19. van Breda HMK, Heesakkers JPFA. Neuromodulation for voiding dysfunction when and how best to use. Curr Bladder Dysfunct Rep 2014;9:41-47.

20. McGuire E, Zhang S, Horwinski E, Lytton B. Treatment of motor and sensory detrusor instability by electrical stimulation. J Urol 1983;129:78-79.

21. Amarenco G, Ismael SS, Even-Schneider A et al. Urodynamic effect of acute transcutaneous posterior tibial nerve stimulation in overactive bladder. J Urol 2003 169:2210-2215. 
22. Andrews BJ, Reynard JM. Transcutaneous posterior tibial nerve stimulation for treatment of detrusor hyperreflexia in spinal cord injury. J Urol 2003;170:926.

23. Tai C, Shen B, Chen M, Wang J, Roppolo JR, de Groat WC. Prolonged poststimulation inhibition of bladder activity induced by tibial nerve stimulation in cats. Am J Physiol Ren Physiol 2011;300:F385-F392.

24. Lombardi G, Mondaini N, Macchiarella A, Cilotti A, Popolo GD. Clinical female sexual outcome after sacral neuromodulation implant for lower urinary tract symptom (LUTS). J Sex Med 2008;5:1411-1417.

25. Gill BC, Swartz MA, Firoozi $F$ et al. Improved sexual and urinary function in women with sacral nerve stimulation. Neuromodulation 2011;14:436-443.

26. Yih JM, Killinger KA, Boura JA, Peters KM. Changes in sexual functioning in women after neuromodulation. J Sex Med 2013;10:2477-2483.

27. Parnell BA, Howard JF, Geller EJ. The effect of sacral neuromodulation on pudendal nerve function and female sexual function. Neurourol Urodyn 2015; 34:456-460.

28. van Balken MR, Verguns $H$, Bemelmans BLH. Sexual functioning in patients with lower urinary tract dysfunction improves after percutaneous tibial nerve stimulation. Int J Impot Res 2006;18:470-475.

29. Gokyildiz S, Beji NK, Yalcin O, Istek A. Effects of percutaneous tibial nerve stimulation therapy on chronic pelvic pain. Gynecol Obstet Invest 2012;73:99-105.

30. Musco S, Serati M, Lombardi G et al. Percutaneous tibial nerve stimulation improves female sexual function in women with overactive bladder syndrome. Sex Med 2016;13:238-242.

31. Coyne KS, Sexton CC, Irwin DE, Kopp ZS, Kelleher CJ, Milsom I. The impact of overactive bladder, incontinence and other lower urinary tract symptoms on quality of life, work productivity, sexuality and emotional well-being in men and women: results from the EPIC study. BJU Int 2008;101:1388-1395.

32. Salonia A, Zanni G, Nappi RE et al. Sexual dysfunction is common in women with lower urinary tract symptoms and urinary incontinence: results of a cross-sectional study. Eur Urol 2004;45:642-648.

33. Isidori AM, Pozza C, Esposito $\mathrm{K}$ et al. Development and validation of a 6-item version of the female sexual function index (FSFI) as a diagnostic tool for female sexual dysfunction. J Sex Med 2010;7:1139-1146.

34. Worsøe J, Fynne L, Laurberg S, Krogh K, Rijkhoff NJM. The acute effect of dorsa genital nerve stimulation on rectal wall properties in patients with idiopathic faecal incontinence. Color Dis 2011;13:462-466.

35. Gaziev G, Topazio L, lacovelli V et al. Percutaneous tibial nerve stimulation (PTNS) efficacy in the treatment of lower urinary tract dysfunctions: a systematic review. BMC Urol 2013;13:61.

36. Rosen R, Brown C, Heiman J, Leiblum S. The Female Sexual Function Index (FSFI): a multidimensional self-report instrument for the assessment of female sexual function. J Sex Marital Ther 2000;26:37-41.

37. Ware JE, Sherbourne C. The MOS 36-Item Short-Form Health Survey (SF-36) conceptual framework and item selection. Med Care 1992;30:473-483.

38. Scarpero HM, Fiske J, Xue X, Nitti VW. American urological association symptom index for lower urinary tract symptoms in women: correlation with degree of bother and impact on quality of life. Urology 2003;61:1118-1122.

39. Hurst $\mathrm{H}$, Bolton J. Assessing the clinical significance of change scores recorded on subjective outcome measures. J Manipulative Physiol Ther 2004:27:26-35.

40. Harris PA, Taylor R, Thielke R, Payne J, Gonzalez N, Conde JG. Research Electronic Data Capture (REDCap): a metadata driven methodology and workflow process for providing translational research informatict support. J Biomed Inform 2009;42: 377-381.

41. Wiegel M, Meston C, Rosen R. The Female Sexual Function Index (FSFI): cross-validation and development of clinical cutoff scores. J Sex Marital Ther 2005:31:1-20.
42. Yoong W, Shah P, Dadswell R, Green L. Sustained effectiveness of percutaneous tibial nerve stimulation for overactive bladder syndrome: 2-year follow-up of positive responders. Int Urogynecol J Pelvic Floor Dysfunct 2013;24:795-799.

43. Clayton $A H$, Althof $S E$, Kingsberg $S$ et al. Bremelanotide for female sexual dys functions in premenopausal women: a randomized, placebo-controlled dose-finding trial. Womens Health (Lond Engl) 2016;12:325-337.

44. Signorello D, Seitz CC, Berner $L$ et al. Impact of sacral neuromodulation on female sexual function and his correlation with clinical outcome and quality of life indexes: a monocentric experience. J Sex Med 2011;8:1147-1155.

45. Both S, Paul K, Olaf E. Sexual response in women with type 1 diabetes mellitus: a controlled laboratory study measuring vaginal blood flow and subjective sexual arousal. Arch Sex Behav 2015;44:1573-1587.

46. Cramp JD, Courtois FJ, Ditor DS. Sexuality for women with spinal cord injury. J Sex Marital Ther 2015;41:238-253.

47. Rice IC, Zimmerman LL, Ross SE, Berger MB, Bruns TM. Time-frequency analysis of increases in vaginal blood perfusion elicited by long-duration pudendal neuromodulation in anesthetized rats. Neuromodulation 2017;20:807-815.

48. Zimmerman LL, Rice IC, Berger MB, Bruns TM. Tibial nerve stimulation to drive genital sexual arousal in an anesthetized female rat. J Sex Med 2018;15:296-303.

49. Ammi M, Chautard D, Brassart E, Culty T, Azzouzi AR, Bigot P. Transcutaneous posterior tibial nerve stimulation: evaluation of a therapeutic option in the management of anticholinergic refractory overactive bladder. Int Urogynecol J Pelvic Floor Dysfunct 2014;25:1065-1069.

50. Booth J, Hagen S, McClurg D et al. A feasibility study of transcutaneous posterior tibial nerve stimulation for bladder and bowel dysfunction in elderly adults in residential care. J Am Med Dir Assoc 2013;14:270-274.

\section{COMMENT}

This is an interesting pilot study looking at non-invasive neuromodulation for female sexual dysfunction in women without lower urinary tract conditions. It follows work done years ago, by our group and others, that demonstrated improved sexual function in women undergoing neuromodulation for urologic conditions. However, a drawback of the prior studies was an inability to determine if sexual function improved de novo from neuromodulation or as a result of less bother from the urinary condition that drove patients to seek treatment. The current study shows a clear benefit of neuromodulation to arousal, orgasm and possibly lubrication, opening the door for further investigation into the use of neuromodulation for female sexual dysfunction - a condition that has been challenging to treat with few available therapies.

Bradley Gill, MD, MS Cleveland, $\mathrm{OH}$, USA

Comments not included in the Early View version of this paper. 